Die Charaktertiere bleiben Ren und Mikrofauna. Zu einer Unterscheidung von Tundra- und Steppenphase reicht die Fauna des Kartsteins, wie schon bemerkt, nicht aus.

Eine Umrechnung der Schichtenstärke in absolute Chronologie, wie sie Nuesch am Schweizersbild versuchte, ist wegen der Verschiedenheit der Verwitterungsfaktoren ohne Wert. Sie ergibt in allen Fällen, wo es sich um Eiszeiten und allgemein um rauhe Klimata handelt, viel zu hohe Werte.

Dasselbe Schema, das die Profile der Kartsteinhöhle liefern, folgerte Wiegers 1909 aus den Schichten von Hundisburg bei Magdeburg. Er setzt das Chelleen in das letzte Interglacial, das Acheuleen in die Úbergangszeit zur letzten Eiszeit (diese Zeitschrift I 1909 S. 1 ff.).

Auch R. R. Schmidt kommt durch seine Ausgrabung im Sirgenstein zu einer ähnlichen Aufstellung. Er setzt allerdings den Beginn des Moustérien in die letzte Eiszeit, während im Kartstein der Beginn des Mousterien noch in die Ubergangsperiode - die Primigeniuszeit - des letzten Interglacials fällt. Ein derartiger unbedeutender Zeitunterschied kann aber sehr wohl in den Differenzen zwischen den lokalen Ausbildungsformen des Moustérien seinen Grund haben.

Zum Schluss drängt es mich, an dieser Stelle den Dank allen denen auszusprechen, welche die Ausgrabungen ermöglichten und förderten. Zunächst sei hier genannt Herr Kommerzienramt Bankdirektor Albert Heimann in Cöln, welcher hochherzig die beträchtlichen Mittel zur Verfügung stellte, ebenso die Besitzer des Kartsteins, die Herren Ev. Esser. Sägewerksbesitzer in Eiserfey, und E. van Hoof, Brauereibesitzer in Münstereifel, welche in uneigennütziger Weise die Ausgrabungen gestatteten und die Funde dem städtischen prähistorischen Museum zu Cöln überwiesen.

\title{
Zur Geschichte der Fibel "mit umgeschlagenem Fuss“
}

\section{Von Max Ebert}

Die Fibel mit umgeschlagenem Fuss ${ }^{1}$ ) darf der wichtigste der spätkaiserzeitlichen, germanischen Fibeltypen genannt werden. Sie ist am weitesten verbreitet, und da sie sich im Gegensatz zu andern kaiserzeitlichen Fibelgruppen durch zahlreiche Münzbeifunde nicht nur relativ, sondern auch absolut gut datieren lässt, als ein wertvolles Hilfsmittel für die chronologische Festlegung vieler Funde ihres Verbreitungsgebietes angesehen. Sie ist weiter, wie nachgewiesen, ${ }^{2}$ ) der Prototyp fast aller späteren germanischen und römischen Fibelformen. Eine Ausnahme davon machen

1) Der Terminus stammt von Tischler, Ostpreussische Gräberfelder III in den Schriften der physik.-ökonomischen Gesellschaft zu Königsberg XIX (1878) S. 183.

2) Vgl. O. Almgren, Studien über nordeuropäische Fibelformen der ersten nachchristlichen Jahrhunderte. Stockholm 1897. S. 85 ff. u. a. a. 0. 
nur die Scheibenfibeln. So stammt vor allem von ihr ${ }^{1}$ ) die germanische Bügelfibel der Völkerwanderungszeit, die durch ihre reiche typologische Abwandlung und als Mitträgerin einer sich organisch entwickelnden nationalen Ornamentik ein gutes Leitmittel durch die germanischen Altertümer des frühen Mittelalters geworden ist.

Trotz der besonderen Aufmerksamkeit, die der Fibel m. u. F. von Hildebrand, ${ }^{2}$ ) Tischler, ${ }^{3}$ ) Montelius, ${ }^{4}$ ) Almgren ${ }^{5}$ ) und Salin ${ }^{6}$ ) zuteil wurde, haben bis jetzt diese Fibeln nach Almgrens Ausdruck, ${ }^{7}$ ) etwas Rätselhaftes an sich. Einerseits zeigen sie nämlich eine unverkennbare Ähnlichkeit mit den La-Tène-Fibeln, sowohl in dem Bau des Nadelhalters als vor allem in der eingliedrigen Konstruktion der Feder mit nur vier Windungen und Sehne ohne Hakenbefestigung, eine Konstruktion, die mit dem Ende der La-Tène-Zeit völlig verschwindet. Andererseits war, worüber bis jetzt vollkommene Einigkeit herrschte, die Fibel m. u. F. ausschliesslich der jüngeren römischen Periode zuzuweisen und frühestens ans Ende des zweiten Jahrhunderts n. Chr. Geb. zu setzen.

Da sie aller Wahrscheinlichkeit nach, worauf schon Montelius hingewiesen, in Südrussland entstanden ist, von dort aber keine Spuren der La-Tène-Kultur bekannt waren, zudem eine zeitliche Lücke von etwa 200 Jahren oder mehr klafft, so entschied sich Almgren, der das Problem eingehend behandelte, ${ }^{8}$ ) wenn auch mit einiger Skepsis dahin, dass die Entstehung der Fibel m. u. F. wahrscheinlich nach Südrussland zu verlegen sei und dass die dort gefundenen Stücke offenbar dem Nachlasse der Germanen angehörten. Diese hätten eine noch fortlebende La-Tène-Kultur angetroffen, die Fibelformen aufgenommen und weitergebildet.

Endlich hat Salin zur Frage Stellung genommen. Er leitet die Fibel m. u. F. von einer halbrömischen Form ab, die sich auch in Südrussland gefunden hat. ${ }^{9}$ ) „Die Fibel, Abb. $8,{ }^{10}$ ) hat, von der Seite gesehen, einen äusseren Kontur, der mit dem einer Fibel mit umgeschlagenem Fuss die grösste Ähnlichkeit hat und obendrein sind beide eingliedrig und zeigen dieselbe Konstruktion der Spirale." Die erstere, halbrömische Fibel ist wahrscheinlich aus einem einzigen Stück herausgehämmert, „,eine Arbeit, die eine sehr hohe Fertigkeit in der Metallkunst voraussetzt." Salin glaubt nun, dass die aus einem Drahte hergestellte Fibel m. u. F. eine Nachahmung dieser Fibel ist von Leuten, die über ein geringeres metallurgisches Können

1) Almgren a. a. O. S. 87; Salin, Die altgermanische Thierornamentik 1904 S. 9 ff.

2) Hans Hildebrand, Studier i jämförande fornforskning I. Bidrag till spännets historia in Antiqvarisk tidskrift for Sverige IV (Stockholm 1872-80) Text in 1873.

3) Vgl. Anm. 1.

4) O. Montelius, Den nordiska jernålderens kronologi in Svenska fornm. foren. tidskrift IX (1895 - 96) S. $220 \mathrm{ff}$.

5) Vgl. Anm. 2.

(b) Vgl Anm. 3.

7) Almgren a. a. O. S. 71 .

8) Almgren a. a. O. S. 73.

9) Salin a. a. O. Fig. 8 (= hier Abb. 1).

10) Fig. $8=$ Abb. 1 a.

Praehistorische Zeitschrift III Heft 3/4. 1911 
verfügten und schliesst mit Berücksichtigung des Auftretens der Fibel m. u. F. erst vom Ende des zweiten Jahrhunderts n. Chr. Geb. ab: „da die Fibel m. u. F. später eine gewissermassen alles beherrschende Rolle in der germanischen Welt spielt, kann man kaum die Vermutung unterdrücken, dass das niedriger stehende Volk in diesem Falle Germanen gewesen sind." „,Direkte Belege،“ fügt er hinzu, „für die Richtigkeit dieser meiner Ansicht kann ich nicht bringen, und will deshalb nachdrücklich betonen, dass sie sich lediglich auf einen Eindruck gründet, der sich während meiner Studien in mir festigte."

Ich habe schon früher ${ }^{1}$ ) bei der Besprechung von reicher verzierten Fibeln m. u. F. Bedenken gegen die Ansicht Salins (und auch Almgrens) vorgetragen und, die Annahme einer vorgermanischen Existenz dieser Fibel
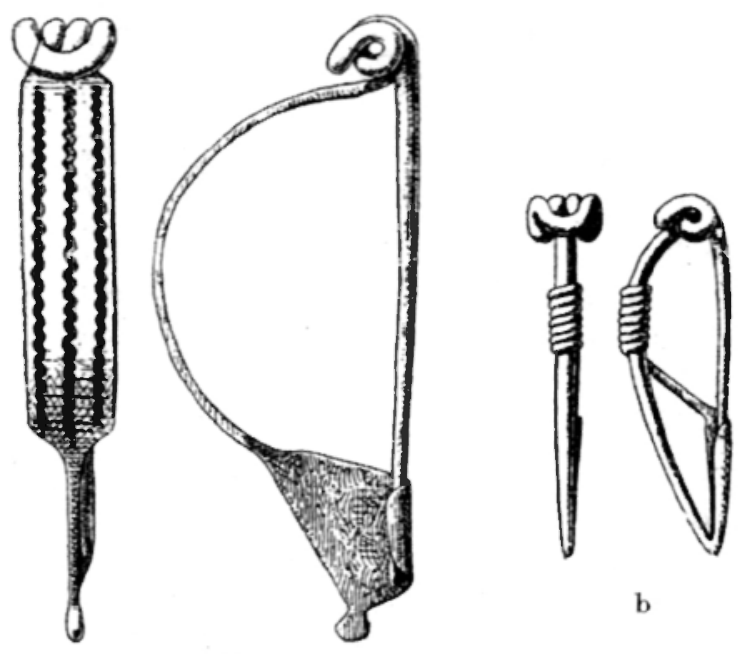

b in Südrussland als notwendig gefordert. Dabei konnte ich mich allerdings nur auf technische und stilistische $\mathrm{Ar}$ gumente stützen. Seitdem ist meines Wissens die Angelegenheit noch nicht wieder diskutiert worden.

Ich glaube aber, dass wir jetzt in der Lage sind, die noch immer offene Frage befriedigend $\mathrm{zu}$ beantworten. Im Frühahre 1911 habe ich im Gouvernement Cherson auf dem Gute Maritzyn (bei Otschakow), etwa $8 \mathrm{~km}$ von Olbia, ein Gräberfeld unter-

sucht, das von der sog. Abb 1. a-b. Bronzene Fibeln von Kertsch. 1/2 nat. Gr.

skythischen Bevölkerung dieser Gegend stammt. Es besteht aus einer Reihe von Kurgangruppen, die eng beieinander liegen, nahe dem Dorfe Petuchofka, am Dnjeprliman. Die meist völlig verschwundenen Grabhügel enthielten etwa 60 Gräber, die teils nischenartig an einen senkrechten Grabschacht sich anschlossen, gegen den sie mit einer Lehmwand abgeschlossen waren, teils von einem Einsteigeschacht aus kammerartig sich erweiterten. In allen Fällen handelt es sich um Skelettbestattung (in Rückenlage). Die Nischengräber enthielten in der Regel nur ein Skelett, die Kammergräber gewöhnlich mehrere.

Im Gegensatz zu einem etwas älteren, im Herbst 1910 von mir untersuchten Grabfeld etwa $1 \frac{1}{2} \mathrm{~km}$ östlich davon, beim Dorfe Adshigol, ${ }^{2}$ ) ist die Ausstattung mit Waffen und wertvolleren Bronzen seltener. Dagegen enthielten die Petuchofkaer Kurgane alle viel keramisches Material, das aus

1) Prähistor. Zeitschr. I (1909) S. $73 \mathrm{ff}$.

2) Die Resultate der Grabungen auf Maritzyn 1910/11 werden in dieser Zeitschrift reröffentlicht. Teil I unten S. $252 \mathrm{ff}$. 
feiner, zum Teil importierter Ware (schwarzgefirniste Vasen, megarische Becher usw.), der einfachen, mit der Scheibe hergestellten Gebrauchskeramik und handgemachten primitiven Gefässen besteht. Dazu kommen Fläschchen aus Alabaster, kleinere Geräte und Schmuckstücke aus Eisen, Bronze, Silber und Gold, Perlen, Korallen und häufig auch Münzen.

Nach Ausweis der Münzen und der übrigen Funde gehören die Kurgane in das vierte bis zweite Jahrhundert vor Chr. Geb., die östlichsten reichen vielleicht ins erste Jahrhundert vor Chr. Geb. hinab.

In zweien nun von den Gräbern, beides Kammergräber der östlichsten Gruppe, haben sich Fibeln gefunden. In dem einen $\left({ }_{3} O\right)$, mit den Skeletten einer erwachsenen Person und zweier Kinder, lag am rechten Unterschenkel des ersten eine bronzene Mittel-La-Tene-Fibel (Abb. 2) mit oberer Sehne, beschädigt, aber in den wesentlichen Teilen erhalten. In dem zweiten Kammergrab $\left({ }_{4} B\right)$, welches fünf Skelette barg, fanden sich beim Skelett III am

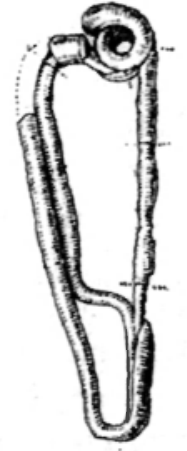

Abb. 2. Bronzene Fibel von Maritzyn (Gouv. Cherson). "/, nat. Gr.

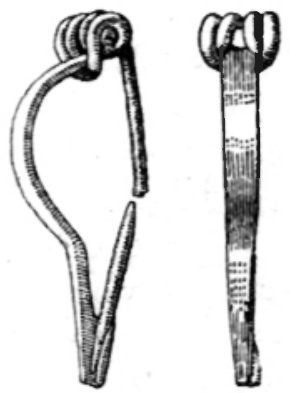

a

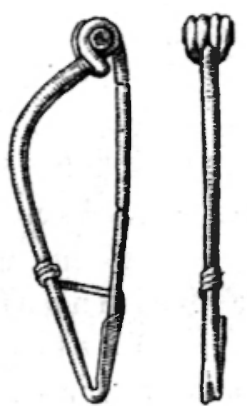

b

Abb. 3. a-b. Bronzene Fibeln von Maritzyn.

(Gouv. Cherson). \%/s nat. Gr.

linken Unterarm eine bronzene Fibel von einem im Westen, soweit mir bekannt, erst in früh-römischer Zeit auftretenden Typus, mit unterer Sehne (Abb. 3a), am linken Oberarm eine bronzene Fibel mit umges chlagenem Fuss (Abb. 3b).

Die Bedeutung dieses Fundes liegt- auf der Hand. Er zeigt zunächst, dass die fraglichen La-Tène-Fibeln am Pontus ungefähr in derselben Zeit auftreten wie in ihrem Entstehungsgebiet in Westeuropa. Ob sie die frühesten Ausstrahlungen der La-Tène-Kultur nach dem Pontus sind, lässt sich vorläufig nicht sagen. Fibeln des Früh-La-Tène-Schemas kommen in Südrussland vor, brauchen aber nicht älter zu sein als die vorliegenden. Die Aufdeckung einer wirklichen La-Tene-Kultur in Südrussland wird kaum zu erwarten sein. Es kann sich hier, wo der hellenistische Einfluss so stark Gegenpart hält, immer nur um vereinzelte Erscheinungen handeln. ${ }^{1}$ ) Vollends

1) Auch die Einwirkung der indigenen, sogenannten skythischen Kultur, die wir noch in Arbeiten der gotischen Epoche zu spüren glauben (de Baye, Mémoires de la Societé nationale des Antiquaires de France. Tome LX 1901) und die schon seit dem Sinken der griechischen Macht vermischt mit orientalischen Zuflüssen kräftiger wird (Sarkophag ron Kertsch, Katakombengemälde, Grabstelen) darf nicht übersehen werden. 
der Nachdauer in die ersten nachchristlichen Jahrhunderte steht nicht nur die griechische Kultur des Pontus, sondern auch die nicht zu unterschätzende römische Einwirkung, die mit den römischen Garnisonen und den ihnen nachfolgenden Händlern festen Fuss im Lande fasst und die immer greifbarer zutage tritt, entgegen.

So sind wir nach unserem jetzigen Wissen berechtigt, anzunehmen, dass die La-Tène-Fibeln, die so vollständig mit den westlichen übereinstimmen, Fremdlinge im Lande und als leicht bewegliche Handelsartikel, wir wissen nicht von welchen Zentren, importiert sind. Im Falle sie stärkeren Absatz fanden, würde freilich die entwickelte Metallfabrikation der griechischen Städte sich wohl dieser Branche bemächtigt haben. Das ist aber kaum der Fall. Zur allgemeinen Ausstattung der Kleidung scheinen sie nicht zu gehören. Klarer sehen werden wir darin jedoch erst, wenn auch diesen Problemen in Südrussland grösseres Interesse zugewendet wird. ${ }^{1}$ )

Der Maritzyner Fund $^{2}$ ) zeigt nun weiter, dass die Fibel m. u. F. in Südrussland älter ist als die germanischen Funde der Kaiserzeit, und ihr Entstehungsgebiet nun wohl mit Sicherheit im Küstengebiet des Pontus gesucht werden muss. Ihr Auftreten in einem unzweifelhaft aus dem 4. bis höchstens 1. Jahrhundert v. Chr. Geb. stammenden Gräberfeld in unmittelbarer Nachbarschaft der Mittel-La-Tene-Fibel erklärt auch die bis jetzt rätselhafte Ähnlichkeit mit diesem Typus.

Die Fibel m. u. F. ist eine Umbildung der Mittel-LaTène-FibelungefährzurZeit vonderenallgemeinem Gebrauch in Westeuropa, in einem Gebiet das vonden Einflüssen der La-Téne-Kultur nur mittelbar, ingeringen A usstrahlungen berührtwird. Sie hat sich in ihrem Entstehungsgebiet, wie es scheint, ohne wesentliche Abänderungen bis zum Einbruche der ostgermanischen Völker gehalten und ist dann von diesen aufgenommen und weitergebildet worden.

Wir beobachten damit auch an den Schicksalen des kleinen, aber für die germanische Welt wichtigen Gebrauchs- und Schmuckstückes in Südrussland einen Zug, der in der Geschichte dieser Kolonialländer immer von neuem hervortritt, die Zähigkeit, mit der sich hier archaische Typen und Formen, Anschauungen, Sitten und Sprache, die im westlichen Mutterland längst durch neue abgelöst sind, unverändert oder nur wenig abgewandelt erhalten haben. E. von Stern ${ }^{3}$ ) hat neuerdings wieder auf den Konservatismus hingewiesen, mit dem die griechischen Kaufleute des 5. und 4. Jahr-

1) Vgl. dazu Almgrens Bemerkung a. a. O. S. 73 Sote 2. Ich werde darauf an anderer Stelle ausführlicher zurückkommen.

2) Bei einer Fibel m. u. F. aus Kurganen bei Olbia (dort mit oberer Sehne), die Pharmakowski 1901 untersucht hat (Iswestia der kais. russ. archaeol. Kommission 19M13 S. $261 \mathrm{Abb}$ 69) sind mir die Fundverhältnisse nicht bekannt: Ph. setzt sie ins $2 / 1$ Jahrh. vor Chr. Geb., was mit unserm Ansatz fïr die Naritzyner Fibel übereinstimmt. Vgl. ferner Iswestia der arch. Kommission 1909 S. 147 n. 29.

3) E. v. Steın, Die gricchische Kolonisation an Nordgestade des Schwarzen Meeres in Klio $\mathrm{IX}(1909)$, S. $147 \mathrm{ff}$. 
hunderts am Schwarzen Meere in der lokalen Kunstarbeit, in Grabanlage, Bestattungsform u. a. an Traditionen des Mutterlandes, die dort längst erloschen waren, festhielten. Wie noch zu Dio Chrysostomos Zeiten die Olbiopoliten auf den Homer schworen. Ein Reisender des 16. Jahrhunderts fand auf der Krim germanisch sprechende Reste der Ostgoten und Heruler (die sog. Krimgoten), ${ }^{1}$ ) und der moderne Besucher Neurusslands wird in den Dörfern deutscher Kolonisten an Sprache und Sitte Schwabens und Sachsens gegen Ende des 18. Jahrhunderts erinnert.

Der konservative Zug des Landes, der aus seiner geographischen Lage und seiner kulturellen Stellung erwächst und sich den verschiedenen, vielfach wechselnden Bevölkerungsschichten mitteilt, führt so zu der in der Geschichte der Fibel nicht häufigen Erscheinung, dass in einem reichen Kulturzentrum sich eines der ,empfindlichsten“ Geräte, das jeder Schwankung des Geschmackes folgt, im wesentlichen unverändert, vier bis fünf Jahrhunderte hindurch erhält.

Die in der Mitte des 3. Jahrhunderts n. Chr. Geb. an den Gestaden des Schwarzen Meeres ankommenden Germanen übernehmen also in geringer Umbildung einen Fibeltypus, den sie ein halbes Jahrtausend vorher in der nordischen Heimat getragen haben. Auch hierin mag eine in nere Erklärung liegen für die dominierende Stellung, die die Fibel mit umgeschlagenem Fuss später unter den germanischen Altertümern einnimmt.

Denn es scheint, dass die Ausbreitung der Fibel m. u. F. bis zur Germaneninvasion sich auf Südrussland beschränkt. Mit der Möglichkeit dass einzelne Stücke schon früher ihren Weg nach dem Norden und Westen finden konnten, muss aber von nun an gerechnet werden. Damit büsst das ist der negative Gewinn unserer Feststellungen - die Fibel m. u. F. zunächst sehr viel von ihrem Ansehen als chronologisches Hilfsmittel ein. Doch wird es vielleicht gelingen, sobald erst grösseres Material aus geschlossenen Funden vorliegt — hier dürfte die nächste Aufgabe weiterer Beschäftigung mit diesem Fibeltypus liegen - leise Wandlungen, die uns bis jetzt entgangen sind, auch in dem langen Ablauf seiner vorgermanischen Geschichte aufzuwersen.

1) R. Löwe, Die Reste der Germanen am Schwarzen Meere, 1896, S. $111 \mathrm{ff}$. 